\title{
Dwarf spheroidal kinematics with large radial velocity samples
}

\author{
Matthew G. Walker $\dagger$ \\ Department of Astronomy, University of Michigan, Ann Arbor, MI 48109, USA \\ email: mgwalker@umich.edu
}

\begin{abstract}
I present a large sample of precise $( \pm 2 \mathrm{~km} / \mathrm{s})$ stellar radial velocities obtained to serve as kinematic tracers in Milky Way dwarf spheroidal (dSph) satellite galaxies. This includes velocities for $\sim 750$ member stars spanning several core radii in the Sculptor dSph, and $\sim 400$ member stars extending out to the nominal tidal radius of the Fornax dSph. The resulting radial velocity dispersion profiles are flat, with no evidence for a sharp decrease in the velocity dispersion at large galactocentric radius in either Sculptor of Fornax. Application of a nonparametric method for estimating the radial mass distributions gives results consistent with mass determinations from classical analyses, with lower limits of $[M / L]_{V} \sim 7$ for Fornax and $[M / L]_{V} \sim 4$ for Sculptor.
\end{abstract}

Keywords. dwarf spheroidal galaxies, dark matter, non-parametric

\section{MMFS: A Powerful New Instrument}

The Michigan MIKE Fiber System (MMFS) is a multi-object echelle spectrograph operational at the twin Magellan 6.5m telescopes of Las Campanas Observatory, Chile. The system consists of two sets of 128 fibers, each accepting light into a 1.4 arcsec aperture and feeding the MIKE spectrograph's two channels (split by a dichroic at $5000 \AA$ ). Both channels of MIKE deliver spectra having $\mathcal{R} \sim 20000$ at high efficiency. The fibers use order-isolating filters to prevent overlap; with the goal of measuring velocities from dSph red giant stars, we use filters that isolate the spectral region near $\mathrm{Mg}$ I and $\mathrm{MgH}$ features at $\sim 5165 \AA$. As both MIKE's channels are sensitive to these wavelengths, we can take spectra of up to 256 objects simultaneously. We find that stars in the magnitude range $V=19.5-20.5$ yield spectra capable of producing precise velocity measurements in 1-3 hours of exposure time. In three observing runs With MMFS we have now collected $>4700$ spectra from $\sim 3500$ stars in the Fornax, Sculptor, Sextans, and Carina dSphs. We are well along the way to our goal of 1000-2000 velocities per dSph.

\section{First Results}

Here I present the first results obtained using MMFS. We have measured $( \pm 2 \mathrm{~km} / \mathrm{s})$ radial velocities for 722 red giants belonging to the Sculptor dSph, and 202 belonging to the Fornax dSph. We combine the Fornax results with previous samples obtained one star at a time (Walker et al. 2005) to arrive at a combined Fornax sample consisting of 357 members. The resulting velocity dispersion profiles of Fornax and Sculptor are shown in Figure 1. Each remains flat over the observed region. The Fornax profile extends to the nominal "tidal" radius, and does not display a sharp decline in velocity dispersion at large radius, as has been found for the Draco and Ursa Minor dSphs (Wilkinson et al., 2004).

$\dagger$ Present address: 830 Dennison Building, Ann Arbor, MI, 48109-1090, USA 

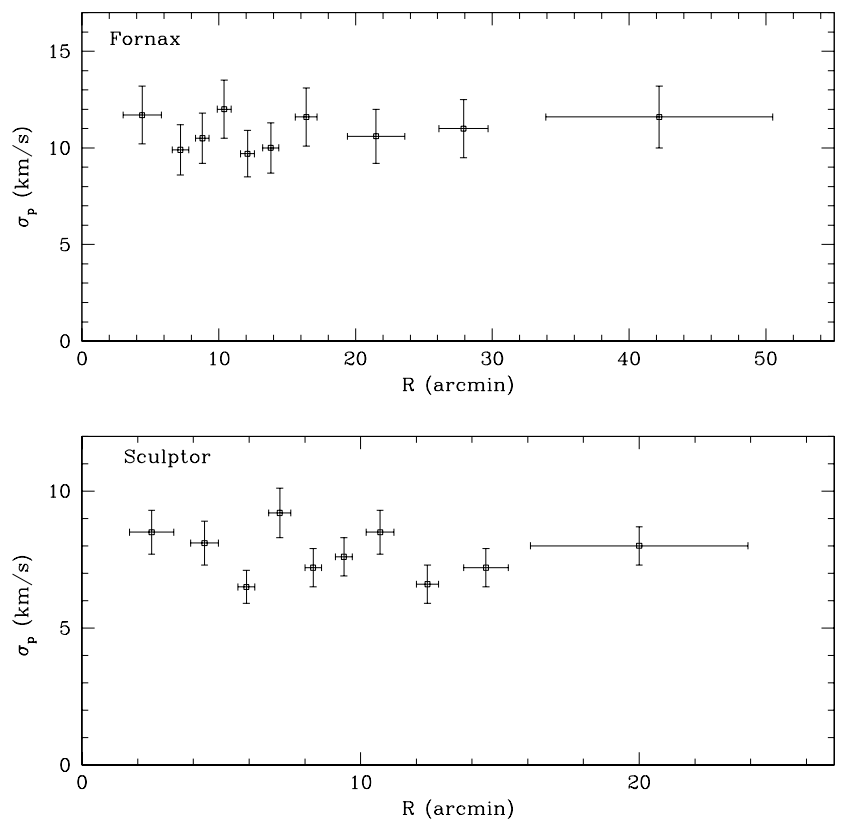

Figure 1. Projected radial velocity dispersion profiles for Fornax and Sculptor. There are 35 stars per bin in the Fornax profile, 72 stars per bin in the Sculptor profile.

\section{A New Nonparametric Mass Estimation Technique}

Wang et al. (2005) introduce a non-parametric method for estimating mass distributions. Implicit are assumptions of dynamic equilibrium, velocity isotropy, and spherical symmetry. Mass is not assumed to follow light, and no stellar distribution function is adopted. One operates directly on photometric data to estimate the deprojected stellar density profile, and then on the radial velocity data to estimate the mass profile. Trials performed on simulated data drawn from a Plummer distribution indicate that samples containing $>1000$ stars are sufficient to estimate $M(r)$ to within a factor of two. Applying this method to the Fornax and Sculptor samples gives $M(r<1500 \mathrm{pc}) \sim 10^{8} M_{\text {sun }}$ for Fornax, and $M(r<400 \mathrm{pc}) \sim 10^{7} M_{\text {sun }}$ for Sculptor, which has only been observed out to a few core radii. Considered with the measured luminosities of these galaxies, these values correspond to lower limits on the M/L of $\sim 7$ for Fornax and $\sim 4.5$ for Sculptor.

\section{Acknowledgements}

I would like to thank my collaborators, Mario Mateo, Edward Olszewski, Michael Woodroofe, and Xiao Wang for outstanding guidance and support.

\section{References}

Mateo, M., Olszewski, E., Welch, D.L., Fischer, P. \& Kunkel, W. 1991, AJ 102, 914

Walker, M.G., Mateo, M., Olszewski, E.W., Wang, X. \& Woodroofe, M. in prep.

Wang, X., Woodroofe, M., Mateo, M. \& Walker, M.G. 2005, ApJ accepted

Wilkinson, M.I., Kleyna, J.T., Evans, N.W., Gilmore, G.F., Irwin, M.J. \& Grebel, E.K. 2004, ApJ 611L, 21 